\title{
An Experimental Evaluation of Broadband Spatial IA for Uncoordinated MIMO-OFDM Systems
}

\author{
J. Fanjul, C. Lameiro, I. Santamaría \\ Department of Communications Engineering \\ University of Cantabria \\ 39005 Santander, Spain \\ Email: $\{$ jacobo,lameiro,nacho\}@gtas.dicom.unican.es
}

\author{
J.A. García-Naya, L. Castedo \\ Department of Electronics and Systems \\ University of A Coruña \\ 15071 A Coruña, Spain \\ Email: \{jagarcia, luis\}@udc.es
}

\begin{abstract}
In this paper we present an experimental study on the performance of spatial Interference Alignment (IA) in broadband indoor wireless local area network scenarios that use Orthogonal Frequency Division Multiplexing (OFDM) according to the IEEE 802.11a physical-layer specifications. Experiments have been carried out using a wireless network testbed made up of six nodes equipped with Multiple-Input MultipleOutput (MIMO) radio interfaces. This setup allows the implementation of a 3-user MIMO interference channel. We have implemented different IA decoding schemes that operate either before or after the Fast Fourier Transform block. IA has been experimentally evaluated comparing both approaches to analyze its performance in synchronous and asynchronous transmissions. Our results indicate that spatial IA performs satisfactorily in practical broadband indoor scenarios in which wireless channels often exhibit relatively large coherence times.
\end{abstract}

Index Terms - Interference alignment; OFDM; WLAN systems; interference channel; MIMO testbed.

\section{INTRODUCTION}

Interference management is a key issue in the design of wireless systems. When several users transmit over the same wireless resources, orthogonal access techniques, such as Frequency-Division or Time-Division Multiple Access (FDMA and TDMA, respectively), are traditionally applied to avoid interference among them. These schemes imply the division of bandwidth and/or time resources among users, hence decreasing the individual data rates. Interference Alignment (IA) has been recently proposed as an alternative method that confines interference signals within half of the signal space at each receiver, allowing each user to simultaneously transmit over the interferencefree subspace [1].

Although there is a large body of literature addressing IA techniques from a theoretical standpoint, there is still lack of experimental results in real scenarios.

The first work that tackled a real-world implementation of IA was presented in $[2$. This work showed that IA is unaffected by frequency offsets or by the use of different modulations. Imperfect time synchronization, however, affects IA, but this issue can be overcome by performing IA at the sample-level.

IA was further evaluated in [3], where the authors conducted an experimental study over measured indoor and outdoor Multiple-Input Multiple-Output (MIMO) Orthogonal Frequency Division Multiplexing (OFDM) channels. They characterized the effect of spatial correlation and subspace distance, and showed that IA is able to achieve the maximum available Degrees of Freedom (DoF) over realistic channels.

In [4] -6] the first aligned over-the-air transmissions were conducted to evaluate spatial-domain IA in a 3 -user interference channel; and in [4], the feasibility of spatial IA over indoor channels and single-carrier transmissions was studied. The 3-user MIMO interference channel with OFDM transmissions is also studied in [6], where the following impairments were identified as an important source of mismatch between the theoretically promised performance of IA and that observed in practice:

- IA is usually studied assuming perfect CSI is available at every node of the network, which never happens in practice.

- Part of the desired signal energy is lost due to spatial collinearity between signal and interference subspaces.

- In theoretical works it is also assumed that the IA precoders and decoders operate at symbol-level, i.e., after frame detection and time/frequency synchronization. In practical systems, however, detection and synchronization have to be performed at sample-level, right after the RF demodulation stages and hence they are affected by interference.

In this paper we focus on the last point, extending our work in [4], [5] to broadband OFDM wireless transmissions. Specifically, we use the IEEE 802.11a Wireless Local Area Network (WLAN) physical-layer standard [7] as a benchmark to evaluate the performance of spatial IA in a 3 -user $2 \times 2$ MIMO-OFDM indoor channel.

\section{Spatial Interference Alignment}

IA is able to exploit the multiple time, frequency and spatial dimensions available in a wireless system. However, the number of required dimensions is considerably less 


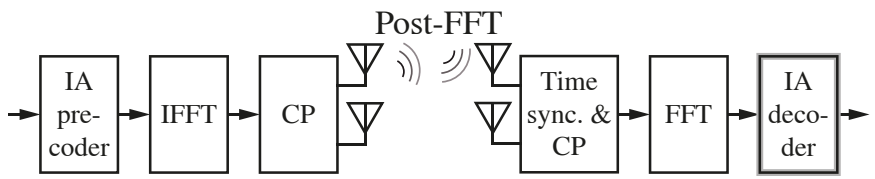

Pre-FFT

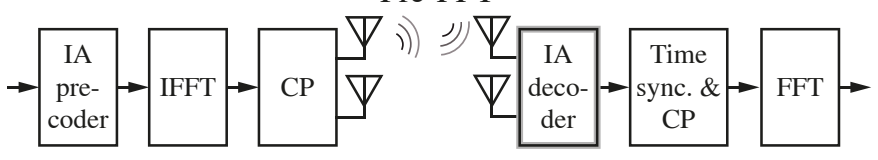

Fig. 1. Post-FFT (up) and pre-FFT (bottom) approaches.

when aligning interference over the spatial dimension [8, 9] which facilitates its practical implementation.

Additionally, depending on the level of coordination among the users participating in the alignment, two different scenarios arise for the application of IA techniques under OFDM packet-based transmissions. In the first scenario, which we denote as synchronous, all users transmit their packets synchronously using the obtained IA precoders. In this case, each receiver can use conventional frame detectors and synchronizers and, consequently, the IA decoder can be applied after the Fast Fourier Transform (FFT) block on a subcarrier basis. In the second scenario, denoted as asynchronous, each user transmits the IA precoded packets at arbitrary time instants. In this situation, if the delay between the received frame coming from the desired user and one of the interfering frames transmitted by the other two users is larger than the Cyclic Prefix (CP) of the OFDM symbols minus the channel delay spread, then conventional frame detectors and synchronizers will fail to work due to the high level of interference at the input of the receiver. In this case, the IA decoder must be applied at sample-level before the FFT (i.e., in the time domain) in order to suppress most of the interference before frame detection is applied. We have implemented both synchronous (post-FFT IA decoding) and asynchronous (pre-FFT IA decoding) schemes, and their pros and cons have been analyzed.

\section{A. Interference Alignment with Post-FFT Decoding}

Let us consider a 3-user MIMO interference channel comprised of three transmitter-receiver pairs (links) that interfere with each other. Each user is equipped with two antennas at both sides of the link and sends a single stream of data. Following the convention introduced in [10, this interference network is denoted as $(2 \times 2,1)^{3}$. Assuming a fully coordinated scenario in which all users transmit their OFDM symbols exactly at the same time instants, or when the possible delays among users can be accommodated by the $\mathrm{CP}$ minus the channel delay spread, each receiver can use a conventional synchronizer and, consequently, the IA decoder can be applied after the FFT block on a carrierby-carrier basis as shown in Fig. 1 (up).
Hence, the decoded signal, $z_{i}$, at the $i$-th receiver for a given subcarrier is ${ }^{1}$

$$
\begin{aligned}
z_{i} & =\mathbf{u}_{i}^{H} \mathbf{H}_{i i} \mathbf{v}_{i} s_{i}+\sum_{j \neq i} \mathbf{u}_{i}^{H} \mathbf{H}_{i j} \mathbf{v}_{j} s_{j}+\mathbf{u}_{i}^{H} \mathbf{n}_{i} \\
& =\mathbf{u}_{i}^{H} \mathbf{H}_{i i} \mathbf{v}_{i} s_{i}+\mathbf{u}_{i}^{H} \mathbf{n}_{i},
\end{aligned}
$$

where $s_{i}$ is the transmitted symbol corresponding to the $i$-th user, $\mathbf{v}_{j}$ and $\mathbf{u}_{i}$ are the precoders and decoders for transmitter $j$ and receiver $i$, respectively; $\mathbf{H}_{i j}$ represents the $2 \times 2$ flat-fading MIMO channel from transmitter $j$ to receiver $i$; and $\mathbf{n}_{i}$ is the additive noise at receiver $i$.

Spatial IA uses a set of precoders, $\left\{\mathbf{v}_{i}\right\}_{i=1}^{K}$, and decoders, $\left\{\mathbf{u}_{i}\right\}_{i=1}^{K}$, that must satisfy the so-called alignment conditions for all transmitter-receiver pairs, $i, j=1,2,3$,

$$
\left\{\begin{array}{l}
\mathbf{u}_{i}^{H} \mathbf{H}_{i i} \mathbf{v}_{i} \neq 0 \quad \forall i \\
\mathbf{u}_{i}^{H} \mathbf{H}_{i j} \mathbf{v}_{j}=0, \quad \forall j \neq i .
\end{array}\right.
$$

In the particular case of the $(2 \times 2,1)^{3}$ interference channel, there is an analytical procedure to calculate the precoders and decoders that satisfy the previous conditions [1].

\section{B. Interference Alignment with Pre-FFT Decoding}

In asynchronous scenarios, the existence of symbol timing offsets between the desired and the interfering OFDM symbols impairs the synchronization procedure. Therefore, in order to reduce interference before the synchronization tasks, pre-FFT IA decoders must be applied at the receiver end. Let us consider a first approach, in which both precoders and decoders are applied in time domain. Also, let $\mathbf{v}_{j}[n]$ be the impulse response of the linear precoder of transmitter $j$ and $\mathbf{u}_{i}[n]$ the impluse response of the preFFT linear decoder of receiver $i$. This leads to a decoded signal at receiver $i$ given by

$$
\begin{aligned}
z_{i}[n]= & \underbrace{\mathbf{u}_{i}^{H}[-n] * \mathbf{H}_{i i}[n] * \mathbf{v}_{i}[n] * x_{i}\left[n-\mu_{i i}\right]}_{\text {desired link }}+ \\
& \underbrace{\sum_{j \neq i} \mathbf{u}_{i}^{H}[-n] * \mathbf{H}_{i j}[n] * \mathbf{v}_{j}[n] * x_{j}\left[n-\mu_{i j}\right]}_{\text {multiuser interference }}+ \\
& \underbrace{\mathbf{u}_{i}^{H}[-n] * \mathbf{n}_{i}[n]}_{\text {noise }},
\end{aligned}
$$

where $x_{j}[n]$ is the discrete-time OFDM signal transmitted by user $j, \mathbf{H}_{i j}[n]$ is the matrix impulse response of the frequency-selective MIMO channel between transmitter $j$ and receiver $i$, and $*$ denotes linear convolution. The received signal at user $i$ is also affected by an additive, spatially and temporally-white Gaussian noise $\mathbf{n}_{i}[n] \sim$ $\mathcal{C N}\left(\mathbf{0}, \sigma^{2} \mathbf{I}\right)$. Notice that we are now considering an asynchronous wireless system and, for this reason, a delay $\mu_{i j}$ between transmitter $j$ and receiver $i$ is explicitly introduced

\footnotetext{
${ }^{1}$ For the sake of conciseness, we have omitted the subcarrier index.
} 


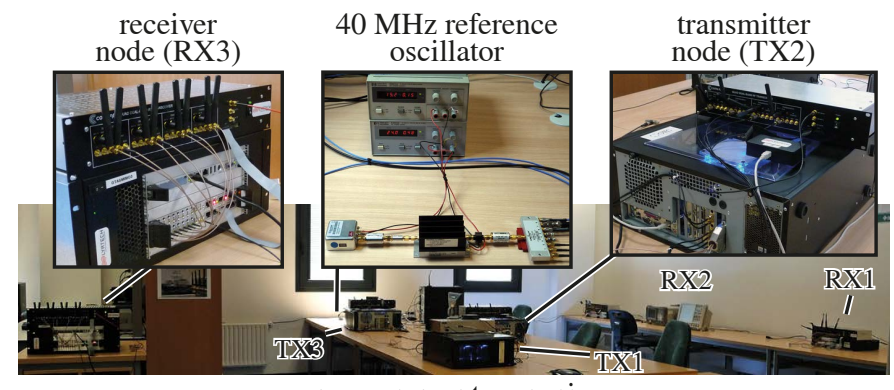

measurement scenario

Fig. 2. Picture of the measurement scenario.

in the signal model given by (3). As shown in [11, the interference leakage after pre-FFT precoding and decoding is given by the sum of the energies of the equivalent interference channels, $\mathbf{u}_{i}^{H}[-n] * \mathbf{H}_{i j}[n] * \mathbf{v}_{j}[n]$ with $i \neq j$. Hence, leakage is independent of the specific delays $\mu_{i j}$ when both precoders and decoders are applied in the time domain. However, it is important to take into account that, for the interference to be mitigated before synchronization, only pre-FFT decoders are strictly necessary, whereas precoders could be applied either in the time or in the frequency domain. Therefore, and for simplicity, we will consider the following approach:

- First, the IA precoders and decoders are computed on a per-subcarrier basis applying the closed-form solution described in [1].

- Next, an $N_{\mathrm{FFT}}$-point IFFT is applied to the set of post-FFT decoders in order to obtain their impulse response.

- Finally, the pre-FFT filters are truncated to a given length, $L$, so as to find the best trade-off between both Inter-Symbol and Inter-Carrier Interference (ISI and ICI, respectively), and Multi-User Interference (MUI) 11.

Notice that the interference leakage turns to be dependent on the delays, $\mu_{i j}$, when the precoders are applied in the frequency domain.

\section{Multiuser Mimo Testbed}

This section describes the MIMO wireless network that has been used to assess, in a realistic scenario, the previously presented IA techniques. The three transmit and receive nodes have a Quad Dual-Band RF frontend, which can use up to eight antennas, connected to four direct-conversion transceivers by means of an antenna switch. Regarding the baseband hardware, each node comprises a VHS-DAC and a VHS-ADC module, respectively, containing eight DAC and eight ADC. Each pair of $\mathrm{DAC} / \mathrm{ADC}$ is connected to a single transceiver in the front-end that admits signals in $\mathrm{I} / \mathrm{Q}$ format.

Figure 2 shows the measurement scenario set up at the University of Cantabria to recreate a typical $(2 \times 2,1)^{3}$ indoor interference channel. The access to the room was carefully controlled during the measurements to ensure that there were no moving objects in the surroundings. Additionally, we also checked that no other wireless system was operating in the $5 \mathrm{GHz}$ frequency band.

\section{A. Measurement Methodology}

Success in the experimental evaluation of wireless communication systems relies mainly on the procedures performed to carry out the measurements. The proposed methodology consists of two stages that require two different over-the-air signal transmissions for the assessment of a single frame per user:

- Training stage: The aim of this phase is to estimate each $2 \times 2$ MIMO channel of the 3 -user interference channel so that the precoding and decoding vectors for each transmission can be computed. For this purpose, all users transmit sequentially (in a TDMA fashion) training frames comprised of $M$ OFDM long training symbols over each antenna, while the three receivers are simultaneously acquiring.

- Data transmission stage: All users transmit simultaneously, hence creating a 3-user interference channel. The IA precoders are applied at the transmitter right before the FFT (frequency domain), and both IA preFFT and post-FFT decoding are performed at the receiver. In a second substage, each user applies the same set of precoders and decoders of the previous scheme but transmitting sequentially in a TDMA fashion, hence avoiding MUI at the receivers. This transmission scheme, which we will denote as Perfect $I A$, allows us to measure the residual interference level created by each transmitter at each receiver in the previous simultaneous phase.

For each channel realization, the foregoing procedure is repeated for all individual data rates specified by the IEEE 802.11a standard.

\section{Experimental Results}

In order to evaluate the performance of the pre-FFT scheme in comparison to post-FFT decoding, we have executed a sufficiently large number of realizations of the aforementioned measurement procedure over different channels. Specifically, binary switches allowed us to use four different two-antenna sets at each node, making a total of 4096 different channel realizations. All channels, estimated by transmitting $M=30$ OFDM long training symbols per training frame, are available for download in the web page of the COMONSENS project [12].

\section{A. Asynchronous Transmission}

We start by studying the performance of pre-FFT and post-FFT IA decoders when users transmit without any coordination. Figure 3 shows the Error Vector Magnitude (EVM) achieved by both schemes. As expected, when there is no coordination among users, post-FFT decoding is not capable of successfully detecting the desired frame due 


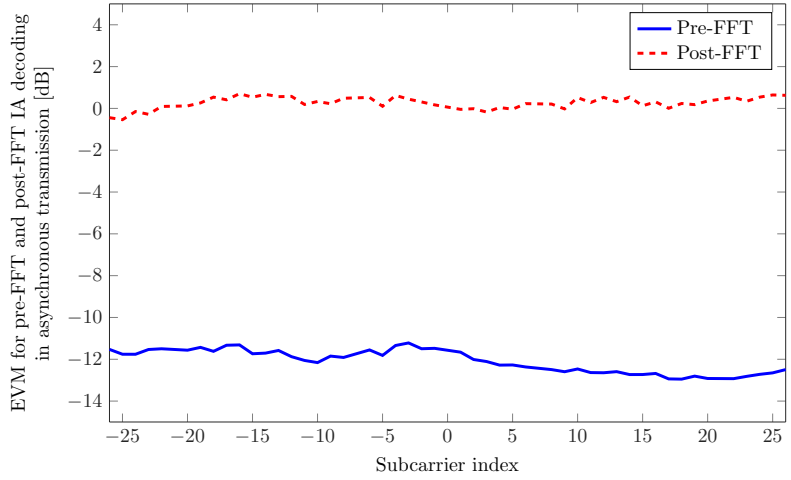

Fig. 3. EVM for pre-FFT and post-FFT IA decoding in asynchronous transmission $[\mathrm{dB}]$.

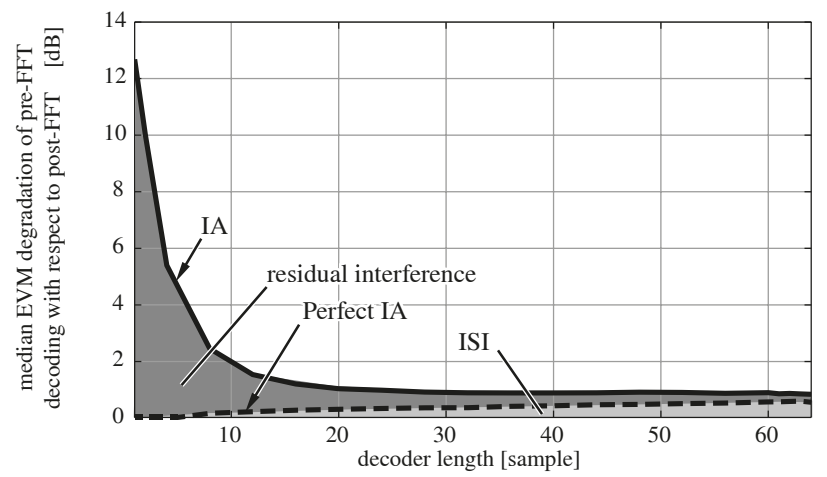

Fig. 4. EVM degradation of pre-FFT decoded transmissions with respect to the post-FFT counterpart.

to the timing offsets and synchronization impairments, as explained in Section II-B

On the contrary, pre-FFT IA decoding overcomes this issue, since synchronization tasks are carried out once the multi-user interference has been successfully supressed at each receiver. Hence, it provides a satisfactory performance, as observed in Fig. 3

\section{B. Synchronous Transmission}

Once we have shown that post-FFT does not work properly when applied to uncoordinated transmissions, we will analyze the degradation of pre-FFT decoding with respect to post-FFT in synchronous scenarios. Let us first study the impact of the pre-FFT decoder length, $L$, on the performance of IA, which, as mentioned in Section II-B involves a trade-off between ISI and residual MUI. To this end, we evaluate the EVM of the received signal constellation for both post- and pre-FFT decoding schemes. Fig. 4 shows the median EVM degradation of the preFFT technique for different decoder lengths, $L \in[1,64]$, with respect to the post-FFT decoder, which obviously provides the best performance. Notice that, for Perfect IA, the degradation is only due to ISI and, as expected, it increases with the decoder length. On the other hand, a

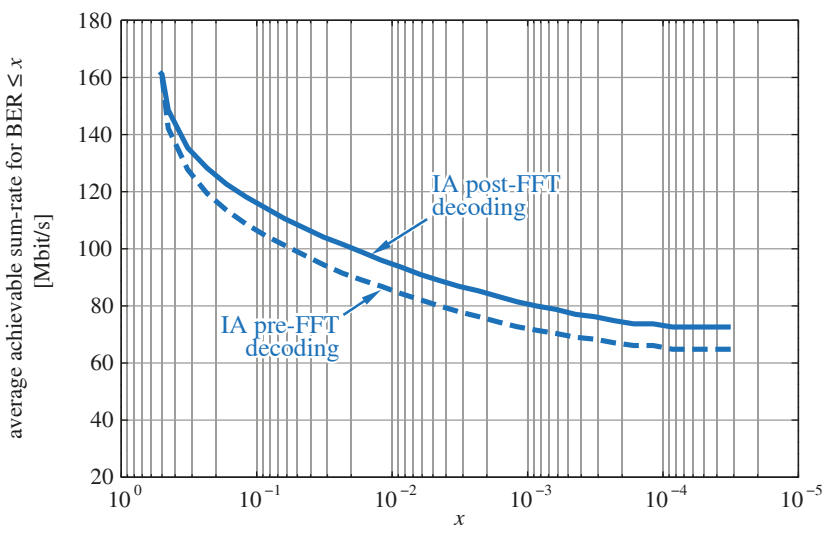

Fig. 5. Average achievable sum-rate that guarantees a given BER for pre-FFT and post-FFT decoding methods.

shortened IA decoder cannot properly suppress the MUI. As the decoder length increases, however, the amount of MUI is reduced whereas the degradation due to ISI grows at the rate seen in the Perfect IA curve.

Finally, in view of the results in Fig. 4, we have chosen an optimal decoder length of $L=30$ samples. Figure 5 represents the average achievable sum-rate that guarantees a Bit Error Rate (BER) equal to or lower than a given value. As expected, post-FFT decoding outperforms the pre-FFT approach in synchronous transmissions. Neverthless, it can be observed that the performance difference is not significant. The pre-FFT decoding scheme has the advantage of being much more robust to time misalignments, hence enabling frame detection in case of a lack of synchronization among users, as seen in Section IV-A. It is also worth pointing out that we have applied a simple approach to obtain the pre-FFT decoders, but more sophisticated algorithms could help reduce the gap between pre- and post-FFT (see [11]).

\section{Conclusion}

In this paper, we have presented an experimental performance evaluation of spatial IA in the 3-user MIMOOFDM interference channel. We have measured received constellation EVM and BER for a set of broadband indoor channels under IEEE 802.11a WLAN transmissions. Our results indicate that pre-FFT (time domain) must be the choice for IA decoding in totally asynchronous scenarios. We have then compared both pre- and post-FFT (frequency domain) in synchronous transmissions, and we have pointed out that the EVM degradation due to pre-FFT approach is less than $1 \mathrm{~dB}$ when choosing an appropriate decoder length.

\section{ACKNOWLEDGMENT}

This work has been supported by the MINECO of Spain and Feder funds of the E.U. under grants CSD2008-00010 (COMONSENS project), TEC2013-47141-C4-R (RACHEL project) and FPU grant AP2010-2189. 


\section{REFERENCES}

[1] V. R. Cadambe and S. A. Jafar, "Interference alignment and degrees of freedom region of the $k$ user interference channel," IEEE Transactions on Information Theory, vol. 54, no. 8, pp. 3425-3441, 2008.

[2] S. Gollakota, S. D. Perli, and D. Katabi, "Interference alignment and cancellation," SIGCOMM Comput. Commun. Rev., vol. 39, no. 4, pp. 159-170, 2009.

[3] O. El Ayach, S. W. Peters, and R. W. Heath, "The feasibility of interference alignment over measured MIMO-OFDM channels," IEEE Transactions on Vehicular Technology, vol. 59, no. 9, pp. 4309-4321, 2010.

[4] Ó. González, D. Ramírez, I. Santamaría, J. A. GarcíaNaya, and L. Castedo, "Experimental validation of interference alignment techniques using a multiuser MIMO testbed," in Proceedings of the International ITG Workshop on Smart Antennas (WSA 2011), Aachen, Germany, 2011.

[5] J. A. García-Naya, L. Castedo, Ó. González, D. Ramírez, and I. Santamaría, "Experimental evaluation of interference alignment under imperfect channel state information," in Proceedings of the 19th European Signal Processing Conference (EUSIPCO 2011), Barcelona, Spain, 2011.

[6] P. Zetterberg and N. Moghadam, "An experimental investigation of SIMO, MIMO, interference alignment (IA) and coordinated multi-point (CoMP)," in Proceedings of 19th International Conference on Systems, Signals and Image Processing (IWSSIP 2012), Vienna, Austria, 2012.

[7] IEEE Standard for Information technologyTelecommunications and information exchange between systems-Local and metropolitan area networks-Specific requirements IEEE Std 802.11 ${ }^{T M}$. 2007 (Revision of IEEE Std 802.11-1999), IEEE.

[8] M. Razaviyayn, G. Lyubeznik, and Z.-Q. Luo, "On the degrees of freedom achievable through interference alignment in a MIMO interference channel," IEEE Transactions on Signal Processing, vol. 60, no. 2, pp. 812-821, 2012.

[9] Ó. González, C. Beltrán, and I. Santamaría, "A feasibility test for linear interference alignment in MIMO channel with constant coefficients," IEEE Transactions on Information Theory, vol. 60, no. 3, pp. 1840-1856, Mar. 2014.

[10] C. M. Yetis, T. Gou, S. A. Jafar, and A. H. Kayran, "On feasibility of interference alignment in MIMO interference networks," IEEE Transactions on Signal Processing, vol. 58, no. 9, pp. 4771-4782, 2010.

[11] C. Lameiro, Ó.González, J. Vía, I. Santamaría, and R. W. Heath, "Pre- and post-FFT interference leakage minimization for MIMO OFDM networks," in Proceedings of the 9th IEEE International Symposium on Wireless Communication Systems (ISWCS 2012), Paris, France, 2012.

[12] COMONSENS: Foundations and Methodologies for Future Communication and Sensor Networks. [Online]. Available: http://www.comonsens.org/index. php?name $=$ demonstrators. 